\title{
Status of the Atlas Calorimeters: their performances after two years of LHC operation and plans for future upgrades
}

\author{
C.A. Solans* \\ On behalf of the ATLAS Collaboration \\ Instituto de Física Corpuscular (UV-CSIC) \\ E-mail: carlos.solans@cern.ch
}

The ATLAS experiment is designed to study the proton-proton collisions produced at the Large Hadron Collider (LHC) at CERN. The ATLAS calorimeter system covers pseudo rapidity $|\eta|<$ 4.9. Following installation in 2004-2006, the calorimeters were commissioned and calibrated prior to first collisions in 2009. Since then, over $10 \mathrm{fb}^{-1}$ of pp data have been collected at both $\sqrt{s}=7 \mathrm{TeV}$ and $\sqrt{s}=8 \mathrm{TeV}$. Results on the calorimeters' operation and performance are presented, including the calibration, the energy and time response stability, uniformity, resolution and absolute scale validation in situ. These results demonstrate that the calorimeters are performing well within the design requirements and are giving reliable input to the physics analyses.

Although LHC data-taking is expected to continue for a number of years, plans are already under way for operation at an instantaneous luminosity about 5 times the original design of $10^{34} \mathrm{~cm}^{-2} \mathrm{~s}^{-1}$, referred to as the High Luminosity LHC (HL-LHC). The calorimeter upgrade involves two phases. In the first, upgrades to the LAr calorimeter electronics will provide more granular information to the trigger system and hence reduce the effects of the high pile-up noise. The second phase will be devoted to the complete replacement of the readout electronics of all calorimeters. A number of proposed solutions are discussed.

36th International Conference on High Energy Physics

4-11 July 2012

Melbourne, Australia

${ }^{*}$ Speaker. 


\section{Introduction}

The ATLAS [1] calorimeters [2, 3] are fully operational since their installation in 2004-2006 and have collected more than $10 \mathrm{fb}^{-1}$ of pp data at $\sqrt{s}=7 \mathrm{TeV}$ in 2010 and 2011, and $\sqrt{s}=8 \mathrm{TeV}$ in 2012. Accurate identification of electromagnetic objects (electrons/photons) are performed by the electromagnetic (EM) calorimeters and properties of hadrons, jets, tau leptons, are measured with the hadronic calorimeters. The extended coverage of the calorimeter system provides very good missing momentum measurements.

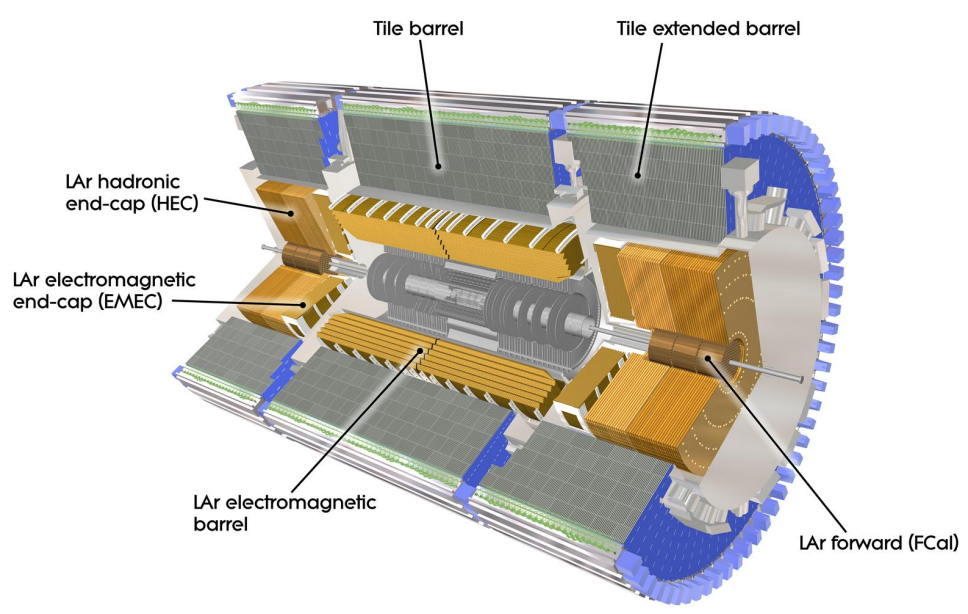

Figure 1: The ATLAS Calorimeters [1].

The ATLAS calorimeters, shown in figure 1, are sampling calorimeters, with liquid argon (LAr) or scintillating tiles (Tile) as active medium. They are composed of 5 different sub-systems that cover different regions of pseudo-rapidity $(\eta)$ and are constructed with different absorber materials. Properties of these various sub-systems are summarized in Table1.

\begin{tabular}{|c|c|c|c|c|l|}
\hline Sub-system & Coverage & $\begin{array}{c}\text { Active } \\
\text { medium }\end{array}$ & Absorber & Channels & $\begin{array}{l}\text { Number of } \\
\text { longitudinal compartments }\end{array}$ \\
\hline EMB & $0<|\eta|<1.475$ & $\mathrm{LAr}$ & $\mathrm{Pb}$ & 109568 & 3 (+presampler) \\
EMEC & $1.375<|\eta|<3.2$ & $\mathrm{LAr}$ & $\mathrm{Pb}$ & 63744 & 3 for $|\eta|<2.5(+$ presampler for $|\eta|<1.8)$ \\
& & & & & 2 for $2.5<|\eta|<3.2$ \\
HEC & $1.5<|\eta|<3.2$ & LAr & $\mathrm{Cu}$ & 5632 & 4 \\
FCal & $0<|\eta|<1.475$ & LAr & $\mathrm{Cu} / \mathrm{W}$ & 3524 & 3 \\
Tile & $0<|\eta|<1.7$ & Plastic & $\mathrm{Steel}$ & 9856 & 3 \\
\hline
\end{tabular}

Table 1: Summary of the characteristics of the ATLAS calorimeters.

In the LAr calorimeters, ionization from charged particles crossing the liquid argon gap in the calorimeters drifts through a high electric field generated by the LAr high-voltage system in between the absorber plates and readout electrodes. This gives rise to a triangle shaped ionization pulse with a steep rise, and slowly falling tail. This analog triangular signal is then processed by the front-end boards, giving a bi-polar pulse further digitized at $40 \mathrm{MHz}$. In the Tile calorimeter the signal deposited in the scintillating tiles is read-out by two photo-multiplier tubes, and the analogue pulse after shaping and amplification is digitized by an ADC at $40 \mathrm{MHz}$. The digital samples are transmitted to the back-end electronics Read-Out Drivers (RODs), where energy and 
time are computed using the Optimal Filtering method shown in equations 1.1.

$$
A=\sum_{i=1}^{N} a_{i}\left(s_{i}-p\right), \quad \tau=\frac{1}{A} \sum_{i=1}^{N} b_{i}\left(s_{i}-p\right)
$$

For each read-out channel, $A$ represents the reconstructed amplitude in ADC counts, which is proportional to the energy in $\mathrm{MeV}$ through a series of constants derived from calibration runs and test beam results [2,3], $s_{i}$ are the digital samples, $N$ the number of samples, 5 in the case of LAr, and 7 in the case of Tile, while $p$ represents the pedestal of each read-out channel. The constants $a_{i}$ and $b_{i}$ are the Optimal Filtering Coefficients, which in the case of Tile, thus sum up to zero. Thus resulting in equations independent of the pedestal value.

The reconstructed time $(\tau)$ is used to filter events for different case studies, like the search of long lived particles or to suppress unwanted backgrounds that might have originated from the neighbouring bunch crossings or from non-collision sources The time resolution at $10 \mathrm{GeV}$ is approximately $0.37 \mathrm{~ns}$ for LAr and $0.8 \mathrm{~ns}$ for Tile, as shown in figures 2, and 3.

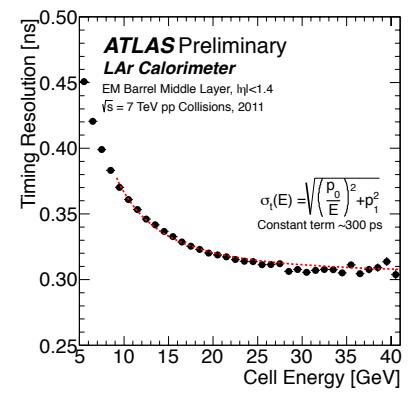

Figure 2: Time resolution from middle layer cells from the LAr EM Barrel Calorimeter [6].

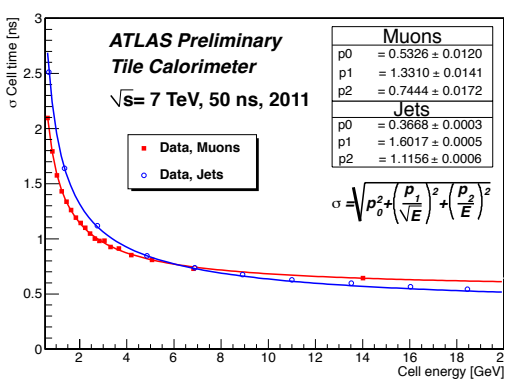

Figure 3: Time resolution from Tile Calorimeter cells using jets or cosmic muon events [5].

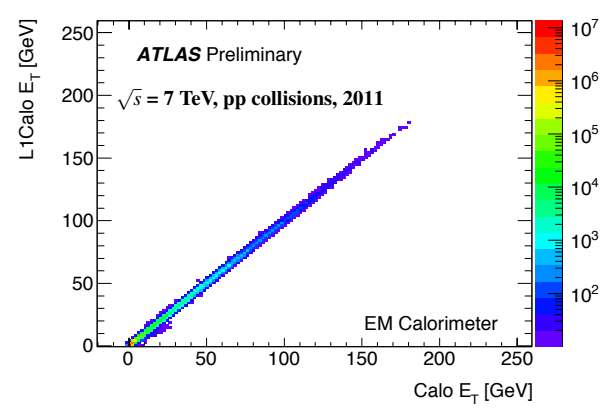

Figure 4: Energy as measured by the L1Calo trigger system vs the calorimeter readout electronics for the EM Calorimeter

In the analogue read-out path, signals are grouped together in trigger towers with granularity of $\Delta \eta \times \Delta \phi \approx 0.1 \times 0.1$ which are provided as input for the Level-1 Calorimeter (L1Calo) trigger. This hardware based trigger has a latency of $2 \mu \mathrm{s}$, and a design resolution of $\sigma_{E_{T}}$ (L1Calo) $<5 \%$ at $E_{T}$ (Calo) $>10 \mathrm{GeV}$. Figure 4 shows a very good correlation between the energy measured by the L1 calorimeter trigger system and the one reconstructed from the LAr readout in the same trigger towers within the expected resolution.

The precision of the calibration constants mentioned in the previous section, are mandatory for the good performance of particle identification and measurements. Figure 5 shows the relative variation in the channel gain, which is smaller than $10^{-3}$, for all LAr sub-systems. Figure 6 shows the deviation from the expected response to a known Cesium radioactive source measured as an average of Tile calorimeter cells with few per mil precision. One can identify the periods of response drifts during collision periods in 2011 and 2012 mainly coming from the photomultiplier response changes due to irradiation, which is partially recovered during periods of no beam activity.

Pile-up is one of the biggest concerns at the LHC, coming both from the same bunch crossing as the triggered one (in time pile-up) or from preceding bunch crossings (out of time pile-up) In time pile-up is observed as an elevation of the cell noise, which increases with the average number 
of interactions per bunch crossing $\mu$. Figure 7 shows the cell noise both from the electronics and the pile-up as a function of $|\eta|$ and calorimeter layer.

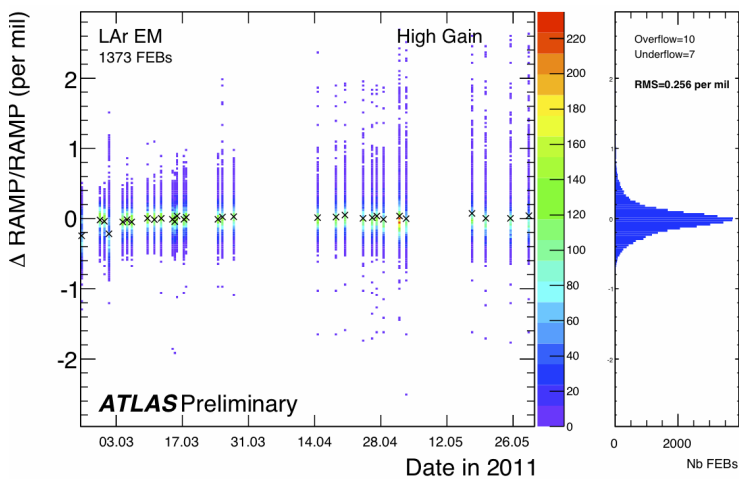

Figure 5: Relative variation in gain per front end board over 3 month period [2]

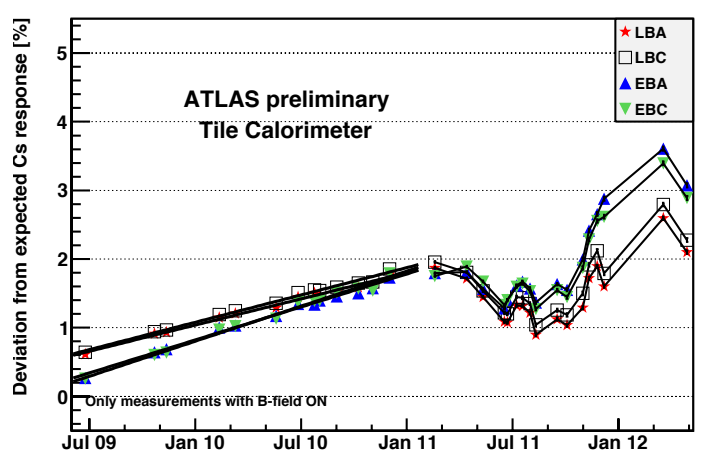

Figure 6: Deviation from the expected Cesium response in the Tile calorimeter [3]

The contribution of the out-of-time pile-up has a different effect. Figure 8 shows the Bunch Crossing Identifier dependence of the isolation energy, deposited in a cone of $\eta, \phi$ of 0.4 around EM objects (removing the energy core). In a train of bunches, constructive interference happens at initial bunches, but after that, the pile-up energy contributions from earlier bunches fully cancel out due to the long tail of the read-out pulse, making the mean of the measured isolation energy independent from the event bunch position in the train. This energy shift is corrected at signal reconstruction level.

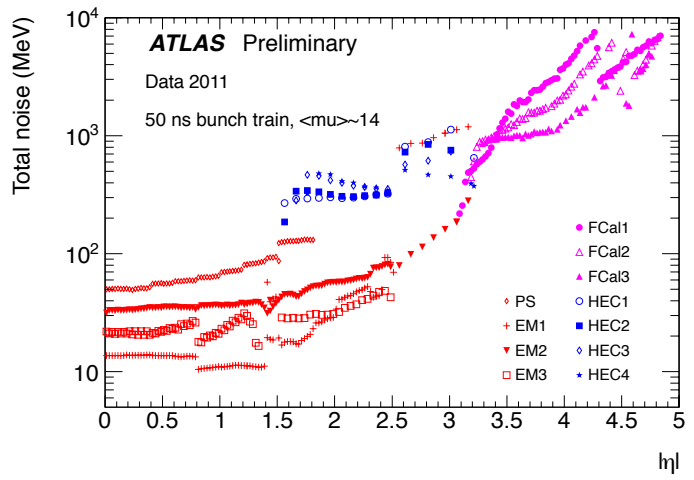

Figure 7: Cell noise accross the different calorimeters as a function of $\eta$, for $\mu=14$ [2].

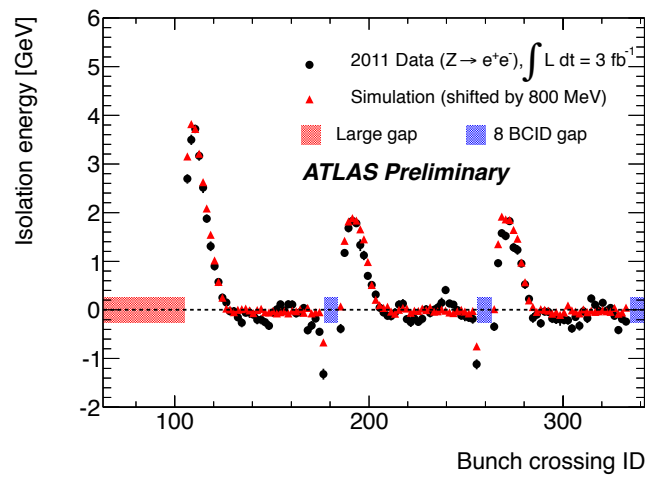

Figure 8: Isolation energy versus Bunch crossing ID [4].

\section{Physics Performance}

High quality physics results put to the calorimeters the following performance goals: precisely calibrated absolute energy scale, linear response over a broad range of energies both for electromagnetic and hadronic objects as well as very high reconstruction efficiency. This is crucial for physics analysis such as the search for Standard Model Higgs decaying into to photon pairs. The results from the ATLAS physics program over the past 2 years of operations strongly vouches for the excellent performance of the ATLAS calorimeters. Figures 9 and 10 show that the calorimeter 
response is very good over a wide range of electron energies. While the electrons from the $J / \psi$ decay have relatively low energy compared to the much harder spectrum of the Z-boson di-electrons, the agreement with the Monte Carlo simulation is excellent in both cases.

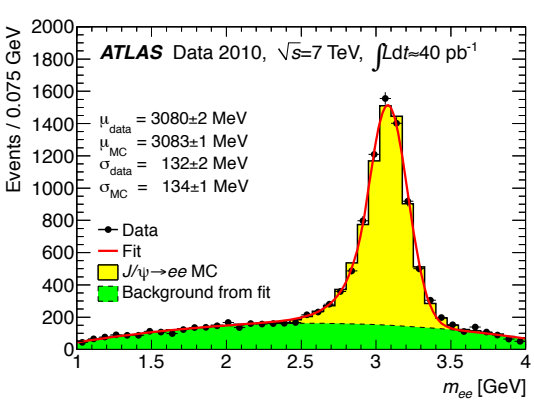

Figure 9: Invariant mass spectrum for electron pairs around the $3 \mathrm{GeV}$ mass window, showing a clear $J / \psi$ peak [7]

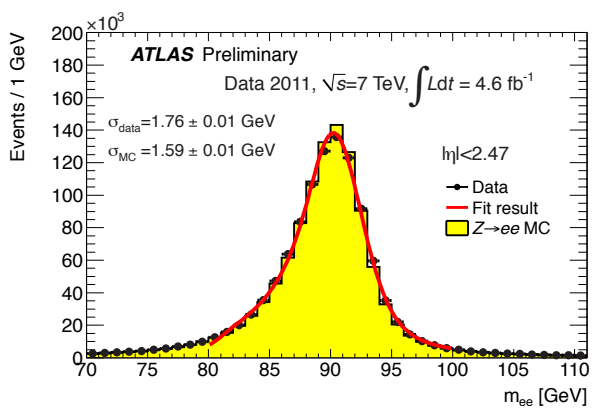

Figure 10: Invariant mass spectrum for electron pairs around $90 \mathrm{GeV}$, showing a clear Z-boson resonance [4].

The electron identification efficiency improved in 2012 providing stable efficiency versus the number of primary vertices as shown in figure 11. Other improvements allow to recover electron candidates that suffered large energy losses due to Bremsstrahlung emissions [4].

Finally, figure 12 shows the fractional jet energy resolution as a function of the average jet transverse momenta measured with the bisector technique in events with two jets in the same rapidity bin for EM+JES calibration with 2011 (red) and 2011 (black) data. It shows a fair agreement between the two years of data taking, with a slight deterioration in 2011 for low $p_{T}$ jets due to the significant pile-up increase.

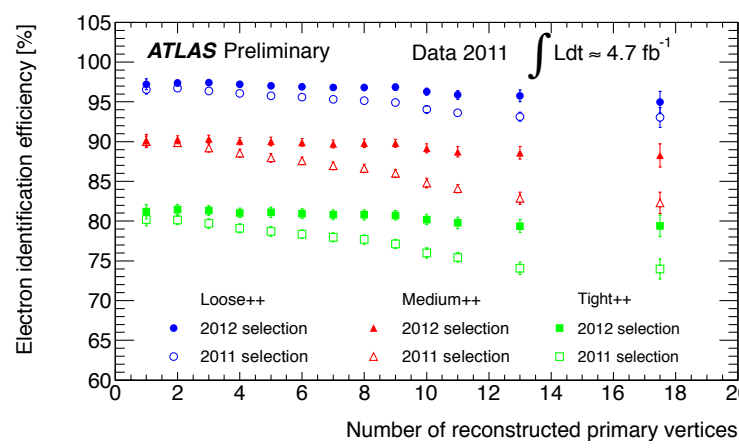

Figure 11: Different electron identification efficiencies vs number of reconstructed primary vertices.

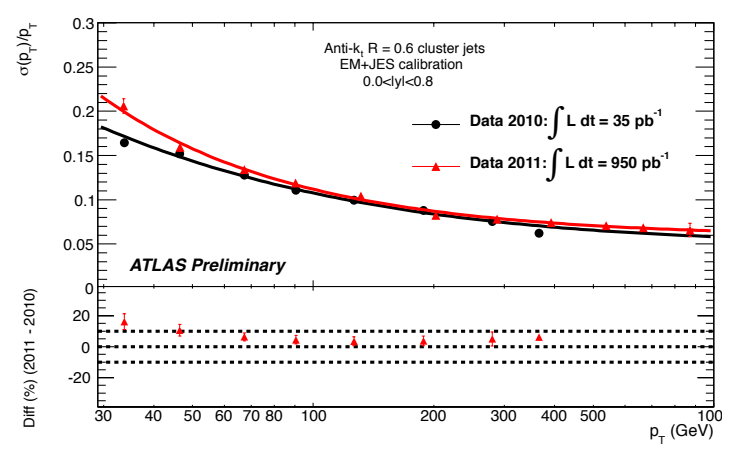

Figure 12: Fractional jet energy resolution as a function of the average jet transverse momenta [7].

\section{Upgrades}

Upgrade plans of the LHC to the HL-LHC [8] foresees an increase of the instantaneous luminosity from $10^{34} \mathrm{~cm}^{-2} \mathrm{~s}^{-1}$ up to $5-7 \times 10^{34} \mathrm{~cm}^{-2} \mathrm{~s}^{-1}$ in two Phases (I and II).

In Phase I, upgrades are required to keep the EM calorimeter trigger rates acceptable at Level 1 with the increase of pile-up noise due to the rise in instantaneous luminosity. These consist of replacing the first and middle Layer Summation Boards (LSB) for the LAr calorimeter, that provide analog sums of $\Delta \eta \times \Delta \phi=0.1 \times 0.1$ by ones that provide increased granularity down to 
$\Delta \eta \times \Delta \phi=0.025 \times 0.1$. This information will be digitized by the LAr Trigger Digitizer Board (LTDB) modules and sent via optical links to the Level-1 calorimeter trigger system. Studies on the sampling frequency, dynamic range, and effective number of bits are in progress. To keep the compatibility with the current system, the intermediate LTDBs will build analog sums as input for the existing Trigger Builder Boards that provide analog inputs to the L1Calo.

The Phase II will be devoted to the complete replacement of the read-out electronics for all calorimeters, where the Tile PMT signals will be digitized continuously at $40 \mathrm{MHz}$, and high speed links will transmit all the samples to off-detector pipelines (super-RODs) that will provide the information to the Level $0 / 1$ trigger. The $R \& D$ for the new system is in progress. Additionally, the increased ionization load poses a number of problems for the FCal that may degrade its performance. These include beam heating, space charge effects in the LAr-gaps, and HV losses due to increased current draws through the current-limiting resistors. There is a proposed design of a copper-diamond calorimeter, which shows a radiation dependent response. The alternative is the replacement of the first layer of the FCal with a new detector with $100 \mu \mathrm{m}$ gaps instead of $250 \mu \mathrm{m}$ ones. However the first option is preferred due to cost and feasibility for the construction and installation of the second one.

\section{Conclusions}

The ATLAS calorimeters are performing very well during the first years of LHC operation. We have a better understanding of the detector, as shown by the recent improvements in particle identification. While the impact of pile-up conditions are significant at the detector level several techniques have mitigated its effect keeping the consequences on data quality at a minor level. The plans for future upgrades target different phases of the HL-LHC, first providing an increased trigger granularity and secondly a major upgrade of the front-end electronics in Tile and LAr calorimeters, where even the FCal hardware will need to be replaced.

\section{References}

[1] ATLAS Collaboration, The ATLAS experiment at the CERN Large Hadron Collider, JINST 3 (2008) S08003

[2] ATLAS Collaboration, Readiness of the ATLAS Liquid Argon Calorimeter for LHC Collisions, Eur. Phys. J. C 70 (2010) 723

[3] ATLAS Collaboration, Readiness of the ATLAS Tile Calorimeter for LHC Collisions, Eur. Phys. J. C 70 (2010) 1193

[4] ATLAS Collaboration, ATLAS experiment Electron/Gamma public results, https://twiki.cern.ch/twiki/bin/view/AtlasPublic/ElectronGammaPublicCollisionResults

[5] ATLAS Collaboration, ATLAS experiment Tile Calorimeter public results, https://twiki.cern.ch/twiki/bin/view/AtlasPublic/ApprovedPlotsTile

[6] ATLAS Collaboration, ATLAS experiment Liquid-Argon Calorimeter public results, https://twiki.cern.ch/twiki/bin/view/AtlasPublic/ApprovedPlotsLAr

[7] ATLAS Collaboration, ATLAS experiment jet/MissingEt public results, https://twiki.cern.ch/twiki/bin/view/AtlasPublic/JetEtMissPublicCollisionResults

[8] ATLAS Collaboration, Letter of Intent Phase I Upgrade, CERN-LHCC-2011-012 